\title{
Adaptive Multi-state Pipe Framework Based on Set Pair Analysis
}

\author{
Leixin Shi, Hongji Xu, Beibei Zhang, Xiaojie Sun, Juan Li, and Shidi Fan
}

\begin{abstract}
Human Activity Recognition (HAR) is one of the main research fields in pattern recognition. In recent years, machine learning and deep learning have played important roles in Artificial Intelligence (AI) fields, and are proven to be very successful in classification tasks of HAR. However, there are two drawbacks of the mainstream frameworks: 1) all inputs are processed with the same parameters, which would cause the framework to incorrectly assign an unrealistic label to the object; 2) these frameworks lack generality in different application scenarios. In this paper, an adaptive multi-state pipe framework based on Set Pair Analysis (SPA) is presented, where pipes are mainly divided into three kinds of types: main pipe, sub-pipe and fusion pipe. In the main pipe, the input of classification tasks is preprocessed by SPA to obtain the Membership Belief Matrix (MBM). The sub-pipe shunt processing is performed according to the membership belief. The results are merged through the fusion pipe in the end. To test the performance of the proposed framework, we attempt to find the best configuration set that yields the optimal performance and evaluate the effectiveness of the new approach on the popular benchmark dataset WISDM. Experimental results demonstrate that the proposed framework can get the good performance by achieving a result of $1.4 \%$ test error.
\end{abstract}

Index Terms-Human activity recognition (HAR), membership belief matrix (MBM), multi-state pipe framework, set pair analysis (SPA).

\section{INTRODUCTION}

Human Activity Recognition (HAR) is an important area in pattern recognition and its research can be traced back to the 1990s [1]. There are two main ways to acquire human activities, i.e., using cameras and wearable sensors [2]. With the rapid development of microelectronics and the Internet of Things (IoT), wearable sensors allow people to interact with devices. Many scholars have made great achievements in using various advanced algorithms to classify and process perceived activity information [3]-[5]. However, there are two drawbacks of the mainstream frameworks: most of them fail to use a divide-and-conquer-based method for different activities and lack generality in different application scenarios.

Manuscript received December 15, 2019; revised January 25, 2020.

This work was financially supported by the National Key Research and Development Program of China (2018YFC0831001, 2017YFC0803403), the National Natural Science Foundation of China (61771292), and the Natural Science Foundation of Shandong Province of China (ZR2016FM29). (Corresponding author: Hongji $\mathrm{Xu}$.)

The authors are with the School of Information Science and Engineering, Shandong University, Qingdao, China (e-mail: sduslx@163.com, hongjixu@sdu.edu.cn).
In this paper, a novel framework is presented for HAR, which utilizes a divide-and-conquer-based method based on Set Pair Analysis (SPA). SPA is a theoretical tool for dealing with uncertainties proposed by K. Q. Zhao [6]. The main idea is that there are two connected sets $A$ and $B$ to form the set pair $H(A, B)$ in a specific problem, which is used to analyze the certainty and uncertainty of the two sets [7]. In the proposed framework, the sample data is pre-classified by K-Nearest Neighbor (KNN), and the results are processed by SPA to obtain the Membership Belief Matrix (MBM). The Membership Belief (MB) is determined by the threshold method for the selection of sub-pipes. Finally, the results are output through the fusion pipe. During the feedback phase, this paper traverses the optimal solution for finding MB to further improve activity recognition performance.

The rest of this paper is organized as follows. The related outline of SPA is introduced in Section II. The details of the proposed framework are described in Section III. The experimental results are showed and analyzed in Section IV. Finally, the conclusions and some future works are described in Section V.

\section{RELATED WORKS}

Some related concepts about existing frameworks of HAR and SPA are reviewed in this section briefly.

\section{A. Framework of HAR}

With the development of hardware processing ability and Artificial Intelligence (AI), the framework of HAR is constantly optimized. The articles [8-11] used various methods of machine learning to identify human behaviors and analyzed the performance of various methods. I. Cleland et al. [12] and K. Altun et al. [13] compared the performances of various machine learning classification methods, and the results indicated that Bayesian Decision Making (BDM) generally can get the highest correct classification rate with relatively small computational cost. The above frameworks are only suitable for dealing with low complexity applications. J. B. Yang et al. [14] proposed a system feature learning method of HAR which used a deep Convolutional Neural Network (CNN) to automate feature learning from the raw inputs in a systematic way. F. J. Ordez et al. [15] proposed a general deep activity recognition framework based on convolution and Long Short-term Memory (LSTM) cycle units. In order to improve the shortcomings of the current frameworks, i.e., their failure to take the important spatial hierarchies between features into account, the concept of capsule was first proposed in 2011 by 
G. Hinton [16] and achieved state-of-the-art accuracy on the dataset MNIST in 2017 [17]. T. Turan et al. [18] monitored infant's emotional cry in domestic environments using the capsule network architecture.



Fig. 1. The structure of the adaptive multi-state pipe framework based on SPA.

However, the above frameworks perform the same processing for all kinds of data, resulting in an inability to distinguish behavior. H. Cho et al. [19] applied a divide-and-conquer approach and built a two-stage activity recognition process, where the abstract activities, i.e., dynamic and static activities, were first recognized using a 2-class classifier, and then the individual activities were recognized using two 3-class classifiers. This framework effectively compensates for the shortcomings of the previous frameworks, but still has shortcomings in generality.

\section{B. Set Pair Analysis}

SPA is a theoretical tool for dealing with uncertainty problems. The basic idea is that in the context of a specific problem, there are two connected sets $A, B$ forming a set pair $H(A, B)$ [20].

The two sets $A$ and $B$ are quantitatively described by the identical, contradictory and discrepant analysis according to their characteristics. The set pair $H(A, B)$ has $M$ features, including the identical features, contradictory features and other features. For a specific problem, assuming that the number of identical features is $S$, and then the identity degree of two sets is $S / M$; the number of contradictory features is $P$, and then the contradictory degree is $P / M$; the number of other features is $F=M-S-P$, and then the discrepancy degree is $F / M$. The connection degree of sets $A$ and $B$ can be expressed as:

$$
\mu_{(A-B)}=\frac{S}{M}+\frac{F}{M} I+\frac{P}{M} J,
$$

where $I$ is the coefficient of discrepancy degree and $J$ is the coefficient of contradictory degree, $I \in[-1,1]$, and the value of $J$ is -1 usually. We set $a=S / M, b=F / M$, $c=P / M$, and the range of them is $[0,1]$. Then, equation (1) can be rewritten as:

$$
\mu_{(A-B)}=a+b I+c J
$$

where $a+b+c=1$.

The proximity of two vectors $v_{1}$ and $v_{2}$.can be represented by:

$$
\cos \theta_{12}=\frac{\left(v_{1}, v_{2}\right)}{\left\|v_{1}\right\|_{2} \cdot\left\|v_{2}\right\|_{2}},
$$

where $\left(v_{1}, v_{2}\right)$ is the inner product of the two vectors $v_{1}$ and $v_{2}$, and $\left\|v_{1}\right\|_{2} \cdot\left\|v_{2}\right\|_{2}$ is the 2-norm product of them.

\section{AdAPtive Multi-State PiPe FRAMEWORK BASED ON SPA}

Differentiation of confusing activities is still a difficult problem in HAR. A novel adaptive multi-state pipe framework based on SPA is proposed to effectively identify confusing activities.

\section{A. Proposed Relational Degree of Evidences}

Fig. 1 shows the basic flowchart of the proposed framework, which is mainly composed of main pipe, sub-pipes and fusion pipe. In the main pipe, the training information is processed by the KNN [21] algorithm to obtain the confusion matrix of each predicted value and actual value, and then the SPA algorithm is used to obtain the Belief Matrix (BM). When the test information enters the main pipe again, the MBM is obtained by multiplying the membership with the previous BM, and then the corresponding sub-pipes are selected by the upper and the lower limits of the credibility to classify the information. Finally, the results in each sub-pipe are output through the fusion pipe.

\section{B. Procedure of the Proposed Method}

As shown in Fig. 2, the flowchart of the adaptive multi-state pipe framework based on SPA is as follows:

- Step 1: Activity information is collected through intelligent hardware as input to this framework.

- $\quad$ Step 2: The proposed framework pre-processes the data and detect whether the information is uncertain by using a preset threshold. If there exists uncertainty, the system will execute uncertainty elimination and information standardization, otherwise it will directly execute information standardization. There are three main types of data standardization.

1) Standardization of category-type features using one-hot coding.

2) Standardization of numerical features using normalization.

3) Standardization of ordered features using ordered numerical coding.

After the original data eliminates uncertainty, activity information will have higher credibility. Normalization unifies the scalar to $[0,1]$, which is convenient for calculation and may help the system to find the global optimal solution.

- $\quad$ Step 3: Enter the appeal results into the main pipe of the framework, and use KNN for classification processing to get the confusion matrix. The columns of confusion 
matrix represent the predicted categories of data and the sum of data in each column is the number predicted as a category. The rows represent the real categories of data, and the sum of data in each row is the number of data instances of one category. The diagonal of the confusion matrix represents the same number of instances as the actual prediction, that is, the prediction is correct. The confusion matrix of the main pipe is showed in Table I, where $n$ represents the number of activity types, and Number $r_{i j}$ represents the number that input activity $i$ is identified as output activity $j$.

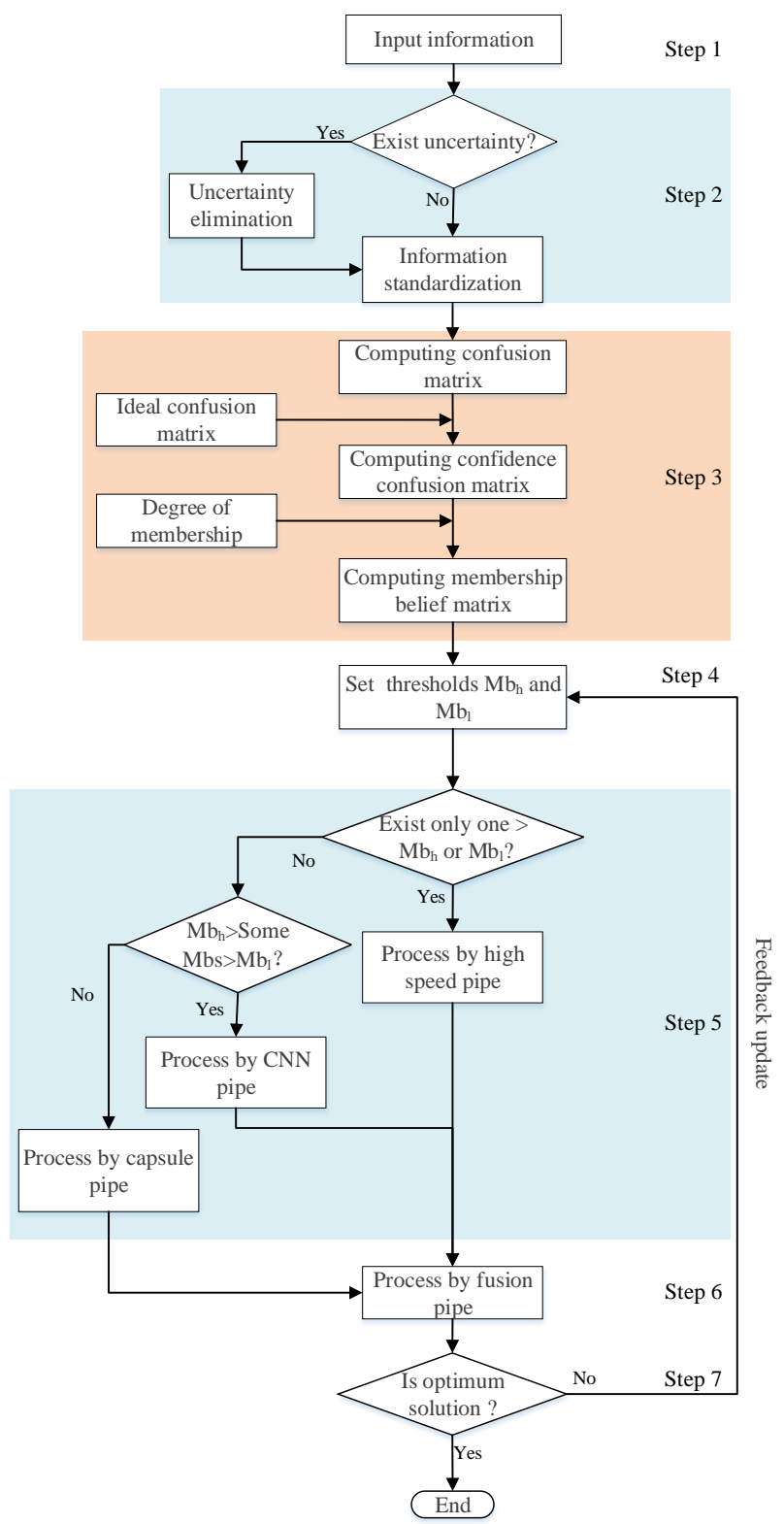

Fig. 2. The flowchart of the adaptive multi-state pipe framework based on SPA.

TABLE I: THE CONFUSION MATRIX OF MAIN PIPE

\begin{tabular}{ccccc}
\hline \hline & Activity 1 & Activity 2 & $\ldots$ & Activity $n$ \\
\hline Activity 1 & Number $_{11}$ & Number $_{12}$ & $\ldots$ & Number $_{1 n}$ \\
Activity 2 & Number $_{21}$ & Number $_{22}$ & $\ldots$ & Number $_{2 n}$ \\
$\ldots$ & $\ldots$ & $\ldots$ & $\ldots$ & $\ldots$ \\
Activity $n$ & Number $_{n 1}$ & Number $_{n 2}$ & $\ldots$ & Number $_{n n}$ \\
\hline \hline
\end{tabular}

TABLE II: THE MEMBERSHIP MATRIX OF MAIN PIPE

\begin{tabular}{cccc}
\hline \hline$v_{1}$ & $v_{2}$ & $\ldots$ & $v_{n}$ \\
\hline$p_{11}$ & $p_{12}$ & $\ldots$ & $p_{1 n}$ \\
$p_{21}$ & $p_{22}$ & $\ldots$ & $p_{2 n}$ \\
$\ldots$ & $\ldots$ & $\ldots$ & $\ldots$ \\
$p_{n 1}$ & $p_{n 2}$ & $\ldots$ & $p_{n n}$ \\
\hline \hline
\end{tabular}

According to the confusion matrix, we can find the membership degree of each predicted activity for the actual activity and express as:

$$
\begin{aligned}
v_{i}=\left[\frac{\operatorname{Number}_{l i}}{\operatorname{sum}\left(\operatorname{col}_{i}\right)},\right. & \left.\frac{\text { Number }_{2 i}}{\operatorname{sum}\left(\operatorname{col}_{i}\right)}, \ldots, \frac{\text { Number }_{j i}}{\operatorname{sum}\left(\operatorname{col}_{i}\right)}, \ldots, \frac{\text { Number }_{n i}}{\operatorname{sum}\left(\operatorname{col}_{i}\right)}\right]^{T} \\
= & {\left[p_{1 i}, p_{2 i}, \ldots, p_{j i}, \ldots, p_{n i}\right]^{T}, }
\end{aligned}
$$

where $p_{j i}$ represents the proportion of actual behavior $j$ in predicted behavior $i, \sum_{k=1}^{n} p_{k i}=1$ and $i \in[1, n]$. Then $\left[v_{1}, v_{2}, \ldots, v_{i}, \ldots, v_{n}\right]$ means the confusion matrix and $\operatorname{sum}\left(\mathrm{col}_{i}\right)$ is the sum of elements in its column $i$.

Table II shows the Membership Matrix (MM) of the main pipe. The ideal membership matrix is a unit matrix, i.e., $\mathbf{E}=\left[e_{1}, e_{2}, \ldots, e_{j}, \ldots, e_{n}\right]$, where $e_{j}=[0,0, \ldots, 1, \ldots, 0]^{T}$. The proximity procedure between the element of actual MM and that of ideal MM is shown as:

$$
\cos \theta_{i j}=\frac{\left(v_{i}, e_{j}\right)}{\left\|v_{i}\right\|_{2} \cdot\left\|e_{j}\right\|_{2}}=\frac{p_{j i}}{\left\|v_{i}\right\|_{2}},
$$

where $\left(v_{i}, e_{j}\right)=\sum_{k=1}^{n} p_{k i} e_{j k}$ represents the inner product of $v_{i}$ and $e_{j},\left\|v_{i}\right\|_{2}=\sqrt{\sum_{k=1}^{n} p_{k i}^{2}}$ represents the 2-norm of the vector $v_{i} \cdot \cos \theta_{i j}$ indicates the proximity of the ideal membership degree of the $i$-th predicted activity and the $j$-th predicted activity. The value of $\cos \theta_{i j}$ is between 0 and 1.

Table III shows the BM of the main pipe, and it indicates that the prediction credibility is $100 \%$ when the element value is 1 .

The membership degree of test information $\mathbf{p}=\left[p_{1}, p_{2}, \ldots, p_{i}, \ldots, p_{n}\right]$ is obtained through the $\mathrm{KNN}$ algorithm of the main pipe, where $p_{i}$ represents the probability that the predicted activity belongs to activity $i$. The membership of the test information and the reliability matrix $\mathrm{BM}$ of the main pipe are multiplied respectively to obtain the MBM, which is shown as:

$$
\mathrm{MBM}=\left[\begin{array}{c}
p_{1} \cos \theta_{11}, p_{2} \cos \theta_{12}, \ldots, p_{i} \cos \theta_{1 i}, \ldots, p_{n} \cos \theta_{1 n} \\
p_{1} \cos \theta_{21}, p_{2} \cos \theta_{22}, \ldots, p_{i} \cos \theta_{2 i}, \ldots, p_{n} \cos \theta_{2 n} \\
\vdots \\
p_{1} \cos \theta_{i 1}, p_{2} \cos \theta_{i 2}, \ldots, p_{i} \cos \theta_{i i}, \ldots, p_{n} \cos \theta_{i n} \\
\vdots \\
p_{1} \cos \theta_{n 1}, p_{2} \cos \theta_{n 2}, \ldots, p_{i} \cos \theta_{n i}, \ldots, p_{n} \cos \theta_{n n}
\end{array}\right],
$$


TABLE III: THE BELIEF MATRIX OF MAIN PIPE

\begin{tabular}{cccc}
\hline $\cos \theta_{11}$ & $\cos \theta_{12}$ & $\ldots$ & $\cos \theta_{1 n}$ \\
$\cos \theta_{21}$ & $\cos \theta_{22}$ & $\ldots$ & $\cos \theta_{2 n}$ \\
$\ldots$ & $\ldots$ & $\ldots$ & $\ldots$ \\
$\cos \theta_{i 1}$ & $\cos \theta_{i 2}$ & $\ldots$ & $\cos \theta_{i n}$ \\
$\ldots$ & $\ldots$ & $\ldots$ & $\ldots$ \\
$\cos \theta_{n 1}$ & $\cos \theta_{n 2}$ & $\ldots$ & $\cos \theta_{n n}$ \\
\hline
\end{tabular}

TABLE IV: ALGORITHM IMPLEMENTATION FOR FINDING OPTIMAL PARAMETERS

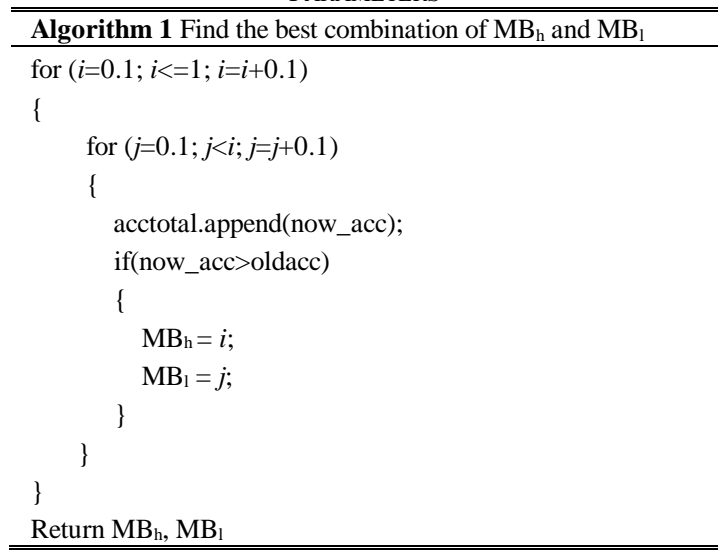

- $\quad$ Step 4: Define a new concept of credibility MB for sub-pipe selection as $\mathrm{MB}_{i j}=p_{i} \cos \theta_{i j}$. The sub-pipe shunt processing is performed according to the upper limit $\left(\mathrm{MB}_{\mathrm{h}}\right)$ and the lower limit $\left(\mathrm{MB}_{\mathrm{l}}\right)$ of the threshold.

$$
\begin{aligned}
& \mathrm{MB}_{\mathrm{h}}=0.8 \times\left(\frac{0.8}{\sqrt{0.8^{2}+(1-0.8)^{2}}}\right)=0.776 \\
& \mathrm{MB}_{1}=0.6 \times\left(\frac{0.6}{\sqrt{0.6^{2}+(1-0.6)^{2}}}\right)=0.498
\end{aligned}
$$

where the membership degree corresponding to $\mathrm{MB}_{\mathrm{h}}$ is initialized to 0.8 and the membership degree of test data is 0.8 . The one corresponding to $\mathrm{MB}_{\mathrm{l}}$ is initialized to 0.6 and the membership degree of test data is 0.6. Initial values of them are determined by simulations, $\mathrm{MB}_{\mathrm{h}}$ and $\mathrm{MB}_{1}$ are then equal to 0.776 and 0.498 , respectively.

- Step 5: The sub-pipes are divided into high-speed sub-pipe, CNN sub-pipe, and capsule sub-pipe. If the classification result of main pipe is accurate, the data passes through the high-speed sub-pipe. Otherwise the sub-pipe using $\mathrm{CNN}$ or capsule is used for secondary classification.

- Step 6: The processed data is sequentially merged through the fusion pipe.

- $\quad$ Step 7: Traverse the sub-pipeline between $\mathrm{MB}_{\mathrm{h}}$ and $\mathrm{MB}_{1}$ in 0.1 step to find the best solution. The results adjust step 4 as feedback. The algorithm implementation to find the optimal $\mathrm{MB}_{\mathrm{h}}$ and $\mathrm{MB}_{\mathrm{l}}$ is shown in Table IV.

\section{EXPERIMENTS AND EVALUATION}

The experiments are performed on a PC equipped with Intel Core i7-8700 + 16G RAM + NVIDIA GeForce GTX
$1080 \mathrm{Ti}$, and run on windows system through Pycharm + Anaconda (Python 3.6.5) + CUDA 9.0 + Cudnn + Tensorflow-gpu 1.8 .

In order to demonstrate the efficiency of the proposed framework, the experiments are performed on the public dataset WISDM. The dataset collects 36 people's activities through sensors in smartphones, including the following six activities: walking, standing, going upstairs, going downstairs, sitting and jogging. The whole data is divided into $75 \%$ training data and $25 \%$ testing data. We analyze how $\mathrm{MB}_{\mathrm{h}}$ and $\mathrm{MB}_{\mathrm{l}}$ of the proposed framework affect the classification results, and confirm the optimal configuration of parameters. Finally, we show the performance of the proposed framework by comparing with some other state-of-the-art frameworks, including $\mathrm{CNN}$ and a two-stage learning of multiple 1D CNN model.

\section{A. Optimal Configuration of $M B$}

Fig. 3 shows the comparison of the accuracy of different thresholds of the adaptive multi-state pipe framework based on SPA. The abscissa represents $\mathrm{MB}_{1}$, the legend represents $\mathrm{MB}_{\mathrm{h}}$, and the ordinate represents the accuracy of different thresholds of the proposed framework. After several simulations we find out that the recognition rate of the framework is optimal when the probability coefficient corresponding to $\mathrm{MB}_{\mathrm{h}}$ is 0.8 and the one corresponding to $\mathrm{MB}_{1}$ is 0.6 . In different application scenarios, the optimal parameters may be different.

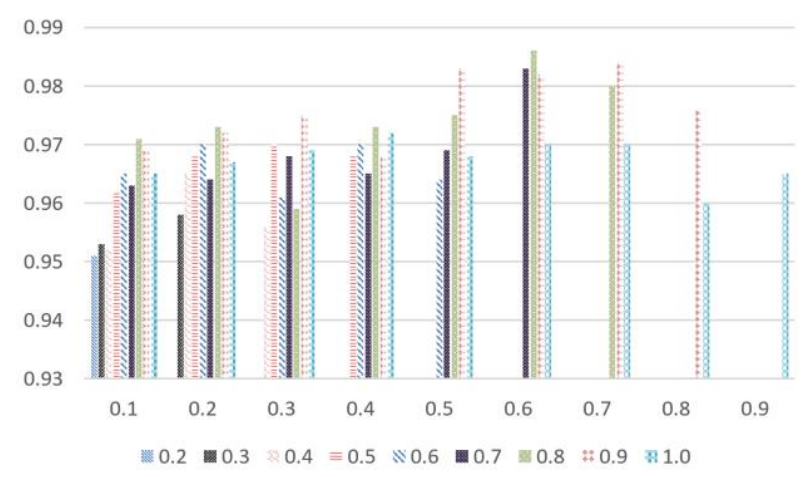

Fig. 3. Comparison of the accuracy of different thresholds of the adaptive multi-state pipe framework based on SPA.

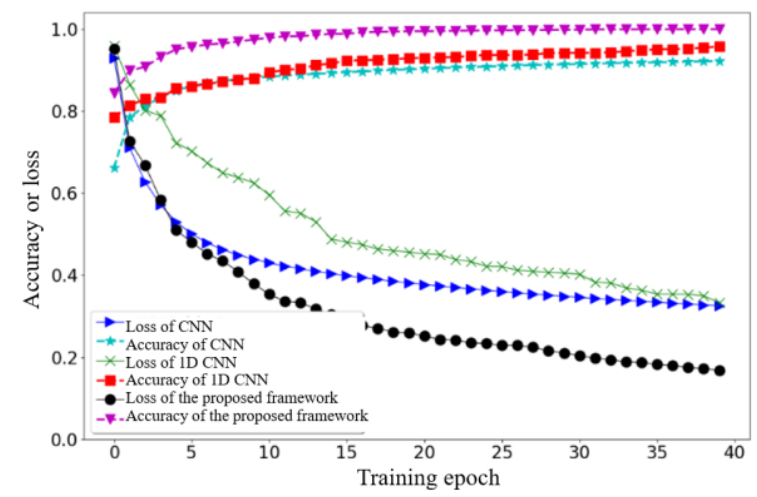

Fig. 4. The accuracy and loss of CNN, 1D CNN and the adaptive multi-state pipe framework based on SPA.

\section{B. Comparison with the State-of-the-Art Frameworks}

In terms of the performance of the proposed framework, 
we compare it with some other state-of-the-art frameworks of HAR. Fig. 4 shows the accuracy and loss of CNN, 1D CNN and the proposed framework. We can see that when the loss tends to converge, the recognition accuracy of the proposed framework is $98.6 \%$ (i.e., the test error is $1.4 \%$ ). During the training process, the recognition accuracy of the proposed framework is always higher than that of CNN or 1D CNN.

Table $\mathrm{V}$ shows comparison of the accuracy of the above three frameworks, we can see that the proposed framework's testing recognition rate is optimal, which is about $7.9 \%$ higher than that of $\mathrm{CNN}$ and $2.2 \%$ higher than that of $1 \mathrm{D}$ CNN.

Fig. 5 shows the accuracy of each activity with the proposed framework. It can be seen that the proposed framework has relatively high recognition accuracy for each activity. Fig. 6 shows the accuracy of each activity of CNN, $1 \mathrm{D} \mathrm{CNN}$ and the proposed framework, respectively. It can be seen that the recognition accuracy of jogging, walking and standing are very high. However, like most other classifiers, $\mathrm{CNN}$ and 1D CNN fail in distinguishing between very similar activities like going upstairs and going downstairs. The proposed adaptive multi-state pipe framework based on SPA can handle these activities more effective and get the best performance.

TABLE V: THE COMPARISON OF THREE FRAMEWORKS

\begin{tabular}{ccc}
\hline \hline Framework & Training Accuracy & Testing Accuracy \\
\hline CNN & $92.3 \%$ & $90.7 \%$ \\
1D CNN & $98.6 \%$ & $96.4 \%$ \\
The proposed framework & $99.9 \%$ & $98.6 \%$ \\
\hline \hline
\end{tabular}

\begin{tabular}{|c|c|c|c|c|c|c|c|}
\hline & Accuracy & Downstairs & Joging & Sitting & Standing & Upstairs & Walking \\
\hline \multirow{4}{*}{ True } & Downstairs & 0.945 & 0.017 & 0.000 & 0.000 & 0.017 & 0.021 \\
\cline { 2 - 8 } Label & Jogging & 0.009 & 0.979 & 0.000 & 0.000 & 0.007 & 0.005 \\
\cline { 2 - 8 } & Sitting & 0.000 & 0.000 & 0.947 & 0.045 & 0.008 & 0.000 \\
\cline { 2 - 8 } & Standing & 0.000 & 0.003 & 0.000 & 0.988 & 0.006 & 0.003 \\
\cline { 2 - 8 } & Upstairs & 0.013 & 0.013 & 0.001 & 0.003 & 0.952 & 0.018 \\
\cline { 2 - 8 } & Walking & 0.005 & 0.001 & 0.000 & 0.000 & 0.006 & 0.988 \\
\hline
\end{tabular}

Fig. 5. The accuracy of each activity.

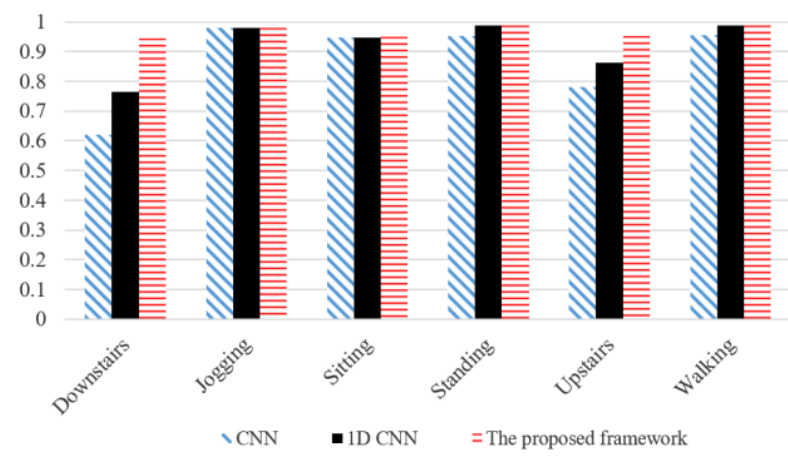

Fig. 6. The accuracy of each activity of CNN, 1D CNN and the adaptive multi-state pipe framework based on SPA.

\section{CONCLUSION}

In this paper, an adaptive multi-state pipe framework based on SPA is proposed, which provides an effective and feasible method for the differentiation of confusing activities.
In the experiments, the optimal configuration of parameters is confirmed by analyzing the impact of several key parameters on the proposed framework. To demonstrate the strength of the proposed framework, it is compared with two state-of-the-art frameworks on the raw time series of the WISDM dataset. The experimental results demonstrate that the proposed framework's recognition accuracy is $98.6 \%$ on the dataset WISDM, which is about $7.9 \%$ higher than CNN and $2.2 \%$ higher than 1D CNN. Therefore, it can be used in both highly confused and general situations, and the fusing results are more effective compared with some other typical frameworks. Future works will consider combining capsule and LSTM to identify the characteristics of human activity.

\section{CONFLICT OF INTEREST}

The authors declare no conflict of interest.

\section{AUTHOR CONTRIBUTIONS}

Leixin Shi conducted the research and wrote the paper; Juan Li and Shidi Fan analyzed the data; Beibei Zhang and Xiaojie Sun assisted with the final draft; Hongji Xu supervised the work and reviewed the paper; all authors had approved the final version.

\section{REFERENCES}

[1] F. Foerster, M. Smeja, and J. Fahrenberg, "Detection of posture and motion by accelerometry: A validation study in ambulatory monitoring," Computers in Human Behavior, vol. 15, no. 5, pp. 571-583, 1999.

[2] O. D. Lara and M. A. Labrador, "A survey on human activity recognition using wearable sensors," IEEE Communications Surveys and Tutorials, vol. 15, no. 3, pp. 1192-1209, 2013.

[3] J. D. Wang, Y. Q. Chen, S. J. Hao, X. H. Peng, and L. S. Hu, "Deep learning for sensor based activity recognition: A survey," Pattern Recognition Letters, vol. 119, no. 5, pp. 3-11, 2019.

[4] A. M. Shaheen, S. A. Raza, and A. Nadeem, "Study of multi-classification of advanced daily life activities on shimmer sensor dataset," International Journal of Communication Networks and Information Security, vol. 8, no. 2, pp. 86-92, 2016.

[5] T. Sztyler, H. Stuckenschmidt, and W. Petrich, "Position-aware activity recognition with wearable devices," Pervasive and Mobile Computing, vol. 38, no. 3, pp. 281-295, 2017.

[6] K. Q. Zhao, "Set pair analysis and its preliminary applications," Nature Exploration, vol. 11, no. 1, pp. 67-72, 1994

[7] K. Q. Zhao, "Disposal and description of uncertainties based on the set pair analysis," Information and Control, vol. 24, no. 3, pp. 163-166, 1995.

[8] Y. Jie, Y. Qiang, and J. J. Pan, "Sensor-based abnormal human-activity detection," IEEE Transactions on Knowledge Data Engineering, vol. 20, no. 8, pp. 1082-1090, 2007.

[9] S. J. Preece, J. Y. Goulermas, L. P. J. Kenney, and D. Howard, “A comparison of feature extraction methods for the classification of dynamic activities from accelerometer data," IEEE Transactions on Biomedical Engineering, vol. 56, no. 3, pp. 871-879, 2009.

[10] A. Mannini, and A. M. Sabatini, "Machine learning methods for classifying human physical activity from on-body accelerometers," Sensors, vol. 10, no. 2, pp. 1154-1175, 2010.

[11] W. C. Cheng, and D. M. Jhan, "Triaxial accelerometer-based fall detection method using a self-constructing cascade-adaBoost SVM classifier," IEEE Journal of Biomedical and Health Informatics, vol. 17, no. 2, pp. 411-419, 2013.

[12] I. Cleland, B. Kikhia, C. Nugent, A. Boytsov, J. Hallberg, K. Synnes, S. McClean, and D. Finlay, "Optimal placement of accelerometers for the detection of everyday activities," Sensors, vol. 13, no. 7, pp. 9183-9200, 2013.

[13] K. Altun, B. Barshan, and O. Tunçel, "Comparative study on classifying human activities with miniature inertial and magnetic sensors," Pattern Recognition, vol. 43, no. 10, pp. 3605-3620, 2010. 
[14] J. B. Yang, M. N. Nguyen, P. P. San, X. L. Li, and S. Krishnaswamy, "Deep convolutional neural networks on multichannel time series for human activity recognition," in Proc. the Twenty-Fourth International Joint Conference on Artificial Intelligence, 2015, vol. 29, no. 6, pp. 3995-4001.

[15] F. J. Ordez and D. Roggen, "Deep convolutional and LSTM recurrent neural networks for multimodal wearable activity recognition," Sensors, vol. 16, no. 1, pp. 159-165, 2016.

[16] G. Hinton, L. Deng, Y. Dong, E. D. George, A. Mohamed, N. Jaitly, A. Senior, V. Vanhoucke, P. Nguyen, T. N. Sainath, and B. Kingsbury, "Deep neural networks for acoustic modeling in speech recognition: The shared views of four research group," IEEE Signal Processing Magazine, vol. 29, no. 6, pp. 82-97, 2012.

[17] S. Sabour, N. Frosst, and G. E. Hinton, "Dynamic routing between capsules," Advances in Neural Information Processing Systems, vol. 29, no. 6, pp. 3856-3866, 2017.

[18] T. Turan, and E. Erzin, "Monitoring infants emotional cry in domestic environments using the capsule network architecture," Proceedings of Interspeech, vol. 29, no. 6, pp. 132-136, 2018.

[19] H. Cho, and S. Yoon, "Divide and conquer-based 1D CNN human activity recognition using test data sharpening," Sensors, vol. 8, no. 4, pp. 1055-1079, 2018.

[20] S. M. Ma, R. C. Wang, and N. Ye, "Reasoning method for context-aware uncertainty based on set pair analysis," Journal of NanJing University of Posts and Telecommunication (Natural Science), vol. 29, no. 1, pp. 64-66, 2009.

[21] S. Sani, N. Wiratunga, S. Massie, and K. Cooper, "KNN sampling for personalised human activity recognition," Case-Based Reasoning Research and Development, vol. 10339, no. 1, pp. 330-344, 2017.

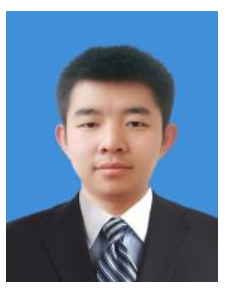

Leixin Shi received the B.S degree in internet of things engineering from Mongolia Industrial University, Hohhot, China, in 2017. He is currently a M.S. student in the School of Information Science and Engineering, Shandong University, Qingdao, China. His research interests include machine learning, artificial intelligence, behavior recognition and ubiquitous computing.

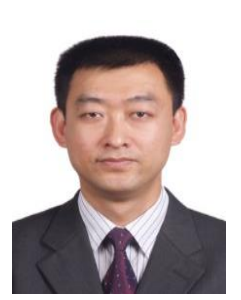

Hongji Xu received the B.S. degree in electronic engineering from Shandong University of Technology, Jinan, China, in 1999, and received the M.S. degree in signal and information processing and the Ph.D. degree in communication and information system from Shandong University, Jinan, China, in 2001 and 2005, respectively. He is currently an associate professor in the School of Information Science and Engineering, Shandong University. From 2004 to 2005, he was a visiting Ph.D. candidate at Telecommunications Technological Center of Catalonia (CTTC) and Department of Signal Theory and Communication, Polytechnic University of Catalonia (UPC), Barcelona, Spain and did research in the areas of wireless communication and signal processing. From 2010 to 2015 , he was a post-doctoral in Tsinghua University - Inspur Group post-doctoral scientific research station, China, and focused on research in multimedia information processing for smart home and cloud computing. From Dec. 2014 to Dec. 2015, he was a visiting scholar in the Department of Cognitive Science, University of California San Diego (UCSD), USA, and did research in the ubiquitous computing and human-computer interaction. From Jan. 2018 to Apr. 2018, he was a visiting scholar in the Virginia Polytechnic Institute and State University (Virginia Tech), USA, and did research in the interdisciplinary fields related to information science and computer science. His research interests include wireless communications, ubiquitous computing, blind signal processing, human-computer interaction and artificial intelligence.

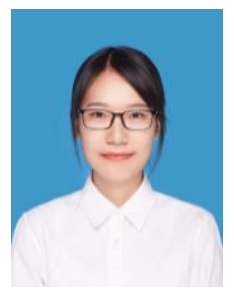

Beibei Zhang received the B.S. degree in communication engineering from Shandong University, Jinan, China, in 2017. She is currently a M.S. student in the School of Information Science and Engineering, Shandong University, Qingdao, China. Her research interests include human activity recognition, data fusion and ubiquitous computing.

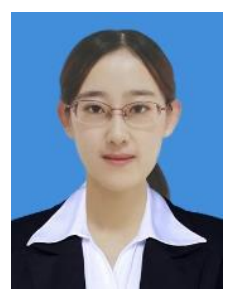

Xiaojie Sun received the B.S. degree in communication engineering from Shandong Normal University, Jinan, China, in 2019. She is currently a M.S. student in the School of Information Science and Engineering, Shandong University, Qingdao, China. Her research interests include machine learning, artificial intelligence, activity recognition and context-aware computing.

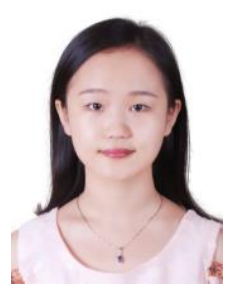

Juan $\mathbf{L i}$ received the B.S. degree in electronic information engineering from Shandong University, Jinan, China, in 2018. She is currently a M.S. student in the School of Information Science and Engineering, Shandong University, Qingdao, China. Her research interests include human activity recognition, data fusion and ubiquitous computing.

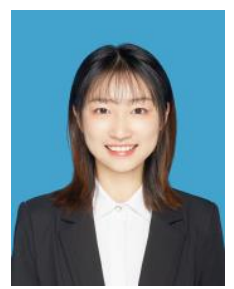

Shidi Fan received the B.S. degree in electronic information engineering from Shandong University, Jinan, China, in 2018. She is currently a M.S. student in the School of Information Science and Engineering, Shandong University, Qingdao, China. Her research interests include artificial intelligence, ubiquitous computing, context awareness, data fusion and quality of context. 